\title{
El crucero transatlántico universitario de 1934 desde un diario inédito
}

\section{The Transatlantic University Cruise of 1934 from an Unpublished Diary}

\author{
MARCELINO JimÉnEZ LEÓN \\ Profesor Serra Húnter de la Universidad de Barcelona \\ marcelino.jimenez@ub.edu
}

Resumen: Este trabajo da cuenta de lo que fue el crucero transatlántico universitario de 1934, organizado por la Universidad de Barcelona (entonces llamada Universidad Autónoma de Barcelona), a partir de una serie de fragmentos del diario inédito que realizó el Dr. Francisco de las Barras y de Aragón con motivo de su participación en este viaje (y que, hasta la fecha, es el único diario conservado sobre el crucero de 1934), así como también de los testimonios epistolares (también inéditos en su mayor parte) de otros cruceristas.

Palabras clave: crucero transatlántico universitario de 1934, diario de viaje, Francisco de las Barras y de Aragón, epistolario.

Summary: This work gives an ccount of what was the 1934 transatlantic university cruise, organized by the University of Barcelona (then called University Autonomous of Barcelona), from a series of fragments of the unpublished travel diary that Dr. Francisco de las Barras y Aragón on the occasion of his participation in this trip (and which, to date, is the only travel diary conserved on the 1934 cruise), as well as the epistolary testimonies (also unpublished for the most part) of other cruise passengers.

Keywords: 1934 transatlantic university cruiser, travel diary, Francisco de las Barras y de Aragón, epistolary. 


\section{CONTEXTO Y PRECEDENTES}

En el corto periodo de tiempo comprendido entre 1933 y 1935 se produce en la universidad española una especie de «frenesí crucerístico» en el que debemos enmarcar el crucero con destino a América organizado por la Universidad de Barcelona en 1934: en 1933 tiene lugar el de la Universidad de Madrid por el Mediterráneo (Gracia y Fullola 2006); en 1934 el de la Universidad de Valladolid a Grecia (Gracia y Fullola 2006: 338-343) y también el de la Universidad de Barcelona a América; por fin, el de 1935, organizado por esta misma universidad, a Extremo Oriente. Si contamos los que se proyectaron pero no llegaron a ejecutarse, entonces debemos añadir uno más: el que estaba previsto que realizaran los estudiantes de Puerto Rico a España en 1936, del que conservamos una mención en una carta de Ángel Ferrer Cagigal a Guillermo Díaz-Plaja en 1935 (AA.VV. 2006: 44-45), una referencia de Guillermo Díaz-Plaja (DíazPlaja 1966: 108) así como parte de la planificación del viaje que he localizado en el Fondo Guillermo Díaz-Plaja (conservado en la Reial Acadèmia de Bones Lletres de Barcelona).

La respuesta a esta profusión de cruceros hay que buscarla en la radical apuesta de la Segunda República por elevar el nivel cultural del país en todos los ámbitos y, más concretamente, en el ministro Fernando de los Ríos, que se esforzó por aplicar los principios de la Institución Libre de Enseñanza a la política educativa española. Los profesores Gracia y Fullola, en las conclusiones a su estudio sobre el crucero de 1933, sintetizaron magníficamente el espíritu del proyecto ministerial:

Es evidente que en el pensamiento de Fernando de los Ríos primaba la idea de sustituir los preceptos más rancios de la docencia, centrados en la clase magistral, por un sistema educativo esencialmente práctico, en el que el conocimiento directo de los temas objeto de estudio y la ampliación de estudios en el extranjero, con la colaboración de la JAE [Junta para la Ampliación de Estudios], permitieran la formación de profesionales que la nueva España republicana necesitaba. La creación en 1932 de la Universidad de Verano en Santander, foro en el que debían impartir docencia los mejores especialistas, es un exponente de su idea renovadora, y la perduración y ampliación del modelo de los cursos de verano, la constatación palmaria de la validez de la idea (Gracia y Fullola 2006: $382)$.

\section{EL CRUCERO TRANSATLÁNTICO UNIVERSITARIO DE 1934}

Pues bien, en el todavía poco trabajado ámbito de los estudios sobre los cruceros universitarios en España, sin duda el más y mejor conocido es el organizado por la Universidad de Madrid en 1933, que cuenta con exposiciones monográficas, libros, estudios, artículos, referencias en semblanzas biográficas y rescate de textos publicados para la ocasión (véase Gracia y Fullola 2006). En 
cambio, no se ha escrito mucho ${ }^{1}$ de una experiencia realizada el verano siguiente, el de 1934, y que fue organizada desde Barcelona y desde su Universidad, entonces llamada Universidad Autónoma de Barcelona y que no hay que confundir con la actual Universidad Autónoma de Barcelona. ${ }^{2}$ Una autonomía recién estrenada y que, desgraciadamente, tendría su primer tropiezo importante pocos meses después. Los hitos de este nuevo viaje sobrepasaban, en cierto modo, los objetivos del crucero precedente. Si el de 1933 fue un crucero eminentemente humanístico, incluso decantado hacia la Historia Antigua, el de 1934 abría nuevos horizontes respecto a varios conceptos. Como, por ejemplo, el concepto de interdisciplinariedad, la coordinación de objetivos docentes, el concepto de interculturalidad (tanto con respecto a los pasajeros como en lo que atañe al itinerario), el establecimiento de relaciones e intercambios con países americanos (que condujeron al proyecto de venida de estudiantes americanos en Barcelona) y la consolidación de esta forma de aprendizaje, con el proyecto posterior —o simultáneo de otros cruceros.

Los cruceros eran, desde luego, un modo diferente, y también usado en otros países, ${ }^{3}$ para ampliar estudios y tomar contacto con la realidad profesional de otros países y continentes, pero, a diferencia de lo sucedido con la Universidad de verano de Santander, esta otra vertiente de la política universitaria republicana no ha tenido continuidad desde 1936, a pesar de los repetidos intentos de Lluís Pericot entre 1953 y 1956 (crucerista de 1933 y catedrático de Prehistoria en la Universidad de Barcelona), según detallan Gracia y Fullola (2006: 344-347).

El crucero fue dirigido por el prestigioso catedrático de Medicina don Ángel Ferrer Cagigal, auxiliado en la organización por dos jóvenes profesores: Guillermo Díaz-Plaja y Jaume Vicens Vives. El objetivo educativo respondía, como en el del 1933, a una política cultural basada en las premisas de la Institución Libre de Enseñanza y en todos los esfuerzos de renovación educativa llevados a cabo durante la Segunda República para conseguir una auténtica

1 Para el crucero de 1934 véanse los trabajos de Díaz-Plaja y Jiménez León (2015), DíazPlaja (2016), Jiménez León (2016a y 2016b) y Merino Escobar (2016), así como la exposición virtual Crucero universitario trasatlántico de 1934: http://crai.ub.edu/es/coneix-el-crai/biblioteques/ biblioteca-lletres/crucero-1934, comisariada por Díaz-Plaja y Jiménez León.

2 La que hoy se conoce como «Universitat de Barcelona» (fundada en 1450) recibió el nombre de «Universidad Autónoma de Barcelona» durante el periodo comprendido entre 1933 y 1939. No debe confundirse con la que actualmente se denomina «Universitat Autònoma de Barcelona», que está ubicada en Bellaterra, pues esta última se creó en 1968. De ahí, que este crucero de 1934 fuese organizado por la Universidad Autónoma de Barcelona, nombre que entonces recibía la actual Universidad de Barcelona.

${ }^{3}$ Fuera de nuestras fronteras, tenemos constancia de un crucero de Marsella a Atenas organizado por el IV Congreso Internacional de Arquitectura Moderna (CIAM) en 1933, al que asistieron un centenar de arquitectos de todo el mundo. Formaban parte de la delegación española «los catalanes Josep Lluís Sert, quien iba acompañado de su mujer Moncha, Josep Torres Calvé, con su hermano músico Raimon, R. Ribas Seva y Antoni Bonet Castellana», y tenía también un marcado carácter transdisciplinar (Biagi 2006: 134); agradezco la información sobre este artículo a Ana Díaz-Plaja. 
universidad renovada en métodos y contenidos, según ya hemos señalado. Conviene subrayar, no obstante, que hay notables diferencias entre el crucero por el Mediterráneo de 1933 y los dos organizados por la Universidad de Barcelona en 1934 y 1935. La primera y principal diferencia es la enorme participación del Estado en el primero, mientras que en los otros dos esta participación es bastante menor, y en cambio está mucho más vinculada a otro personaje clave: el ya citado catedrático de Medicina de la Universidad de Barcelona don Ángel Ferrer Cagigal, cuya figura ha sido hasta la fecha poco estudiada, pero resulta fundamental para entender los dos cruceros organizados por la Universidad Autónoma de Barcelona. Si Ferrer Cagigal fue el factótum en lo que atañe a la iniciativa y las negociaciones con la Compañía Transatlántica (con la que tenía contacto desde la infancia, pues su padre había sido médico de la misma), el «cronista oficial» de ambos fue un viejo amigo de Ferrer: Francisco de las Barras y de Aragón, quien escribió los dos únicos diarios de viaje localizados (hasta la fecha) sobre los cruceros universitarios organizados por la UAB en 1934 y 1935. Conviene, pues, hacer un esbozo biográfico de ambos personajes y de la relación entre ellos.

La mejor semblanza biográfica y profesional de Ferrer Cagigal hasta la fecha es el estudio de Matías-Guiu y Martí Laborda (1981), de donde tomo algunos de los datos expuestos a continuación. Ángel A. Ferrer Cagigal nació en Santander (de donde era oriunda su familia materna) en 1886, pero se trasladó muy temprano a Cádiz, porque su padre era médico de la Compañía Trasatlántica (por vía paterna su familia era catalana y estaba vinculada al mundo de la navegación al menos desde el siglo XVIII, según he podido constatar en la documentación que he localizado en el Arxiu Nacional de Catalunya). Se licenció en Medicina por la Universidad de Cádiz en 1908. Desde muy temprano aparecen en su biografía dos de sus intereses principales: el compromiso social con la Medicina y su afición por los viajes. Con su traslado a la Universidad de Barcelona en 1923, donde gana la cátedra de Histología e Histoquimia y Anatomía Patológica, se inicia para él un nuevo y muy fructífero periodo vital: a partir de aquí tendrán lugar una serie de éxitos profesionales. Entre 1929 y 1931 fue Decano de la Facultad de Medicina, y en 1934 y 1935 se encargó de la dirección de los dos cruceros universitarios (el primero a América y el segundo a Extremo Oriente). Su temprana muerte, en 1936, truncó esta fulgurante trayectoria profesional.

De la otra figura fundamental del crucero de 1934, Francisco de las Barras y de Aragón, hay más bibliografía, ${ }^{4}$ aunque es también un personaje muy interesante sobre el que todavía queda mucho por estudiar. En palabras de Valiente Romero (2013):

representa el modelo de naturalista propio del siglo XIX español que vivió en primera persona la división de la escuela krausista. Sus maestros se adscribían

${ }^{4}$ En especial hay que remitir al estudio de Valiente Romero (2007), aunque, desgraciadamente, el autor no menciona los cruceros de 1934 y 1935, ni el diario que es objeto de este artículo. El primer trabajo sobre este diario inédito es el de Jiménez León 2016a. 
a la facción más progresista, integrándose así Barras en la primera generación de científicos españoles que aceptarán el positivismo. Es la denominada «generación de los grandes sabios» 0 «Edad de Plata de la ciencia española», muy vinculada al regeneracionismo del 98 y cuyo desarrollo se vio truncado con el inicio de la Guerra Civil. Barras fue catedrático de los institutos de segunda enseñanza de Oviedo, Ávila y Huelva, de la Universidad Central, la de Oviedo, Cádiz y Sevilla. [...] La concepción de las Ciencias Naturales durante su formación universitaria era acorde con ese carácter polifacético, abarcando la Botánica, Geología, Zoología y Antropología. En sus más de 300 publicaciones, Barras abordó la totalidad de estas disciplinas, a las que se añade la Historia, que cultivó de forma constante desde el año 1899. No obstante, a partir de 1920, su actividad docente e investigadora se centró fundamentalmente en el marco de la Antropología Física, que simultaneó con la realización de estudios históricos, especialmente de temática científica y americanista.

Un breve repaso por su trayectoria vital muestra, en primer lugar, que hay varios puntos de contacto con la de Ferrer Cagigal: ambos pertenecen a familias acomodadas y en los dos es patente la vinculación familiar con la navegación y en concreto con los viajes a América (véase, para Barras, Valiente Romero 2007: 23-24). Otro aspecto muy interesante del perfil intelectual de Barras, en lo que atañe al espíritu institucionista que está detrás de estos cruceros, es su vinculación con el Krausismo, la Institución Libre de Enseñanza y, más en concreto, con Federico de Castro, figura clave del pensamiento krausista, con cuya hija se casó Barras (vid. Valiente Romero 2007: 38-40 y 103). Por las fechas en que frecuenta este círculo krausista, siendo estudiante, participa también en las excursiones espeleológicas organizadas por Sales y Ferré ${ }^{5}$ (discípulo de Julián Sanz del Río, el introductor del Krausismo en España), «publicando los diarios de muchas de ellas» (Valiente Romero 2007: 35): así pues, su faceta como diarista hunde sus raíces en su época de estudiante.

El hecho de que Francisco de las Barras sea una figura fundamental del crucero de 1934 se debe fundamentalmente a que es el autor del único diario de dicho crucero. Como he señalado en otro lugar (Jiménez León 2016a), el conocimiento de este diario se lo debo al Dr. Daniel Marías Martínez, de la Universidad Carlos III de Madrid, que en la primavera de 2014, durante su colaboración con la Dra. Ana Díaz-Plaja y conmigo en la preparación de la exposición sobre el «Crucero Transatlántico Universitario de 1934», nos informó de su existencia y de su ubicación en el Archivo del Real Jardín Botánico de Madrid. De hecho, la conservación de dichos diarios (y del resto del archivo y biblioteca de Francisco de las Barras) se debe a la diligencia y el sentido previsor de su propietario, quien, consciente del valor patrimonial y con la voluntad de servicio público que le caracterizó toda su vida, repartió su legado entre diversas instituciones públicas, si bien, con el paso del tiempo, parece haberse perdido

5 Para las relaciones entre el Krausismo y Cataluña véase I. Vilafranca (2009) y A. Sotelo Vázquez (2014, en especial las pp. 317-333). 
parte de la documentación (véase Valiente Romero 2007: 119-121). De ahí que en el Archivo del Real Jardín Botánico (CSIC, Madrid) se conserven dos ejemplares mecanografiados de los viajes realizados en 1934 y 1935 (en lo que atañe al primero, el ejemplar lleva el sello de entrada del 6 de marzo de 1952), y en la Biblioteca de la Universidad de Barcelona un ejemplar del viaje de 1935, que es el que se he empleado en este artículo. ${ }^{6}$

\section{DESCRIPCIÓN DEL DIARIO DE 1934 ESCRITO POR F. DE LAS BARRAS Y DE ARAGÓN}

Se trata de un cuaderno mecanografiado con algunas leves correcciones manuscritas del autor. Con una extensión de 85 cuartillas, su redacción es muy sintética, casi diríamos que telegráfica en algunas entradas. Como decía más arriba, a diferencia del crucero de 1933, que generó varios diarios (como consecuencia de la convocatoria de un concurso al respecto), algunos de los cuales han sido editados (entre ellos, y por orden cronológico: Díaz-Plaja, 1935 y 1985; Gracia y Fullola 2006 editaron los de Jaume Vicens Vives, Gregorio Marañón y Esmeralda Gijón Zapata; Esteve Gálvez, 2009 y Marías 2011), en el caso del crucero de 1934 solo se conserva este diario, si bien gracias a este documento (Barras 1934: 83) sabemos que Ferrer Cagigal llevaba un diario manuscrito, y que existía el proyecto de publicar todas las conferencias impartidas durante el crucero «acompañando una relación del viaje», pero el proyecto no se llegó a realizar y tampoco se han podido localizar, hasta la fecha, estos materiales.

El diario ofrece una visión de conjunto de la mano de un experimentado viajero. Por el diario de 1934 sabemos que Barras había estado en Barcelona en 1903 (Barras 1934: 6); en Las Palmas en 1926 (Barras 1934: 29); y en América en 1932, con motivo del aniversario de José Celestino Mutis (Barras 1934: 24). A pesar de su carácter sintético, aporta datos de sumo interés para conocer la gestación, los objetivos y el desarrollo de este crucero. Además, desde la perspectiva del autor, es una especie de «despedida», pues Barras se muestra consciente de que no volverá a pisar esos lugares. Así, escribe en 1934: «pasamos ante la estatua de la Libertad, que aparecía como entre gasas, y no tardó en esfumarse del todo Nueva York, y con él América, que probablemente no veré más» (Barras 1934: 80). ${ }^{7}$

${ }^{6}$ Debo expresar aquí mi agradecimiento a los diferentes archivos y bibliotecas que han hecho posible este trabajo: en primer lugar el Fondo Guillermo Díaz-Plaja (de la Reial Acadèmia de Bones Lletres de Barcelona), el Archivo del Real Jardín Botánico de Madrid (CSIC), el Archivo de la Universitat de Barcelona, el CRAI de la Biblioteca de Letras de esta misma universidad y el Archivo Nacional de Cataluña, por las facilidades que me han prestado en la elaboración de este trabajo y, muy especialmente, al equipo de trabajo del Fondo Guillermo Díaz-Plaja y al Dr. Daniel Marías, de la Universidad Carlos III de Madrid.

7 Nos han llegado tres documentos sobre este crucero de 1934 escritos por Barras. El primero es el diario inédito de 85 páginas, escrito en 1934 y titulado «Primer crucero universitario de Barcelona» (Barras 1934), ejemplar conservado en la Archivo del Real Jardín Botánico (CSIC, 
Procederé, a continuación, a presentar brevemente el contenido de su diario de 1934, haciendo énfasis en los aspectos más relevantes: en primer lugar, debo señalar que ofrece preciosas noticias sobre los diversos aspectos de este interesantísimo proyecto tan poco conocido. Destacaremos algunos, comenzando por el más importante: el que se refiere a la vertiente didáctica del viaje. Por ejemplo, en la primera escala que hace el barco, en Valencia, nos cuenta que Vicens Vives llevó a los alumnos al Museo de Prehistoria de la ciudad, organizado por Pericot (p. 11). Desde el punto de vista educativo, el eje vertebrador eran los ciclos de conferencias: Barras señala la fecha precisa (e incluso la hora, en muchos casos), los temas, su éxito, y las reacciones que suscitaron (véanse, por ejemplo, las pp. 19, 24, 26, 46, 56, 69, 70...). Al hilo de las conferencias tenemos conocimiento de algunas representaciones, como la de la señora Elisa Jiménez de Pérez, quien «por la tarde, había representado admirablemente el gracioso monólogo titulado $\mathrm{Fea}$. Creo que fue esta misma señora la que el día anterior había bailado admirablemente bailes populares de Puerto Rico. Entre ellos un tango de negros» (p. 25). Gracias al diario sabemos de otras actividades culturales: Barras organizó a bordo una especie de taller de medidas antropológicas que tuvo bastante éxito (pp. 22-23 y 83-84), y asistía también a las tertulias (p. 24). Y, sobre todo, nos da cumplida noticia de las visitas formativas que se realizan a universidades, centros sanitarios o compañías petrolíferas (pp. 32, $43,47,50,61,62,64 \ldots)$

Nos informa también de diversos incidentes, vinculados al siempre espinoso tema del nacionalismo y a las peculiares circunstancias políticas de algunos de los países visitados (véanse como ejemplo las pp. 29, 37, 40, 59-60 o 66-67). De ahí que en las comidas y brindis Ferrer Cagigal tenga que emplearse a fondo como mediador: «hubo una sesión de discursos, en la que volvió Ferrer a tener que hacer equilibrios siempre por causa de las estridencias políticas de los oradores» (p. 63; algo similar podemos hallar en las pp. 67 y 68). El contacto con la realidad más mísera aparece en contadísimas ocasiones (véanse en especial las pp. 57-59).

Obviamente, también hay información sobre las fiestas, la vida a bordo, los pasajeros (destaca, por ejemplo, la presencia del embajador Emilio Zapico Zarraluqui, p. 24) y otros muchos detalles de la travesía. Finalmente, el diario sirve también para mostrar la personalidad de su autor: su crítica al mundo yankee (p. 28), su interés por la fotografía, los recuerdos de infancia, los viajes que había realizado previamente o las amistades que había trabado en viajes anteriores, y que ahora reencuentra (como el rector de la Universidad de Caracas, P.D. Rodríguez Rivero, Luis de Oteyza o Clemente García Morales, pp. 38 y 40). Todo ello en un estilo sencillo y muy sintético, casi lacónico.

\footnotetext{
Madrid), bajo la signatura 143/5 (inédito); el segundo es la conferencia de 24 páginas que pronunció en la Sociedad Geográfica, el 11 de marzo de 1935 (el ejemplar mecanografiado se conserva en el Archivo de la Universidad de Barcelona) (Barras 1935a), y el tercero es la publicación de esta conferencia en el Boletín de la Sociedad Geográfica Nacional en junio 1935 (Barras 1935b), tal y como advierte el propio autor en la nota al pie de la p. 323. He realizado el cotejo entre el ejemplar mecanografiado y la publicación, llegando a la conclusión de que son prácticamente idénticos, salvo alguna leve modificación.
} 
Tras esta somera descripción, veamos a continuación en qué consistió el crucero transatlántico de 1934 a partir del testimonio contrapunteado del diario de F. de las Barras y el epistolario de otros pasajeros del crucero (lo cual nos permite contrastar sus informaciones).

\section{LOS PREPARATIVOS DEL VIAJE}

Con el apoyo de la Universidad, los dos organizadores visitaron al Conde de Güell, presidente de la Compañía Transatlántica. El acuerdo fue claro: la expedición universitaria ocuparía una parte importante del pasaje - casi un centenar, sobre un total de 374 viajeros- del vapor Marqués de Comillas, y seguiría la ruta atlántica trazada por este barco. Güiell se comprometió a ofrecer un precio reducido asequible para los estudiantes y profesionales interesados en el crucero. El precio fue de 1.875 pesetas en primera clase, y 1.400 en clase turista.

Así lo explica Guillermo Díaz-Plaja en Memoria de una generación destruida (1966: 108):

Ofrecimos convertir el barco en una Universidad flotante, en la cual se irían pronunciando conferencias sobre temas históricos, literarios o científicos que sirviesen - a universitarios y pasajeros - de ilustración al viaje. Don Juan Antonio accedió y nos ofreció el «Marqués de Comillas»—por cincuenta días de navegación y dentro del tratamiento señorial de la compañía- en unas condiciones verdaderamente mínimas. Dudo que alcanzasen a compensar la mantequilla que se servía en las comidas.

La Universidad ofreció becas y medias becas y promocionó el viaje entre los estudiantes universitarios y el público intelectual interesado. El periódico de Barcelona La Vanguardia iba recogiendo los avisos enviados desde la universidad para preparar documentación de los futuros cruceristas. Empezaron a llegar solicitudes de todo el país: Vigo, Madrid, Valencia, Bilbao... y de los ámbitos académicos y las profesiones más diversas.

De las Barras da cuenta de ello en la primera página de su Diario: ${ }^{8}$

$\mathrm{Al}$ ir al despedirme del Rector me dijo que, puesto que era aficionado a viajes, tenía una buena ocasión aquel verano, y me dio el prospecto que le habían enviado del Primer crucero que se organizaba en la Universidad de Barcelona, y que iba dirigir y había sido su iniciador don Ángel Ferrer Cagigal [...]. No lo pensé mucho tiempo y desde luego que me decidí a escribir a la Universidad de Barcelona diciendo que contaran conmigo [...]. (Barras 1934: 1)

El importe de 1.875 pesetas todo comprendido resultaba una verdadera ganga.

(Barras 1934: 5)

${ }^{8}$ En la transcripción del diario que realizo en este trabajo modernizo la ortografía y la puntuación. 
En este sentido, es muy interesante leer la descripción del crucero que se hace desde la Universidad Autónoma. De las posibilidades que ofrece cada puerto se destacan no únicamente las académicas y culturales, según se puede leer en el folleto editado:

San Juan de Puerto Rico, la perla de las Antillas, donde se podrá visitar su Universidad, Escuela de Medicina, Tropical, centrales azucareras, y admirar toda la belleza de su paisaje tropical, con los fértiles campos dedicados al cultivo de café, caña de azúcar, tabaco y todo tipo de frutas tropicales. ${ }^{9}$

Como se puede comprobar, un interesante programa para animar a estudiantes y licenciados en muchas áreas. La Universidad Autónoma se lanzó a la aventura sin muchos apoyos oficiales. Finalmente se llegó a los 95 inscritos. Un vistazo a la lista de pasajeros confeccionada por la Universidad Autónoma de Barcelona da idea del alcance del viaje y de la intención interdisciplinaria - e interprofesional - que lo animaba. Había estudiantes de Letras en las ramas de Historia, de Filología y de Pedagogía. En un rápido repaso podemos destacar los nombres de Eduard Valentí Fiol y Santiago Sobrequés, además de los mencionados Díaz-Plaja y Vicens Vives. Encontramos también antropólogos, como el Dr. De las Barras de Aragón, o arabistas, como García de Linares. En cuanto al campo científico, abundan los estudiantes o profesionales relacionados con el campo de la sanidad: farmacia, química, y, sobre todo Medicina. En este campo hay que destacar la presencia de unos apellidos que formaban parte de una estirpe de médicos de relieve como los Gallart o los Corachán. Es curiosa también la participación de un pequeño grupo de la Escuela de Ingenieros, o de un grupo de abogados. Además, había muchos profesionales del mundo de la enseñanza: catedráticos de secundaria, maestros del Instituto Escuela y otros docentes. Pero conviene recordar que no se trataba únicamente de un viaje que podía interesar a futuros profesionales en formación, sino que iniciaba una forma de viaje cultural y de aprendizaje que atraía a profesionales en ejercicio que querían aprovechar la oportunidad de una expedición pensada desde y para la ciencia.

El itinerario fue el siguiente: Barcelona-Valencia-Málaga-Cádiz-Canarias. Después, atravesaron el Atlántico hasta llegar a San Juan de Puerto Rico; Sto. Domingo, La Guayra y Puerto Cabello en Venezuela; Curaçao; Puerto Colombia, en Colombia; Colón, en Panamá; Puerto Barrios en Guatemala, La Habana, Cuba y Nueva York en Estados Unidos. En total: 50 días de navegación desde el 20 de julio al 10 de septiembre. El recorrido es, además de extenso, de una variedad riquísima para conocer realidades culturales y científicas muy diversas y cambiantes. Lógicamente, había que aprovechar tantas oportunidades como pudiera ser posible, y por ello, antes de salir, los dos organizadores - Vicens y Díaz-Plaja - ya tenían apalabradas conferencias en universidades y contactos oficiales - y oficiosos - con diversas instituciones de prestigio de cada puerto visitado.

${ }^{9}$ El folleto se conserva, al igual que todos los testimonios epistolares citados en adelante, en el Fondo Guillermo Díaz-Plaja. 
Además, en esta expedición confluían varios aspectos que sin duda alguna levantaban expectación tanto en España como en los puertos de destino. Por un lado, la fuerza renovadora de una universidad que se replanteaba con principios democráticos y modernos; Cataluña estaba desarrollando su Estatuto y unas perspectivas políticas esperanzadoras. También un gobierno estatal en su tercer año de República, lleno de proyectos educativos en la línea de los más prestigiosos movimientos pedagógicos del momento. Y por último, se contaba con el entusiasmo del director Ferrer Cagigal y el empuje de los dos organizadores de Letras. No es de extrañar que en muchos de los países visitados se recibiera el buque Marqués de Comillas como una embajada cultural, científica y política de primer orden. El director, los organizadores y los pasajeros eran conscientes de ello, como atestigua la documentación conservada.

\section{LA VIDA EN EL BARCO}

La idea de un crucero universitario no comporta únicamente los puertos de destino sino también las actividades de formación a bordo. Los profesores Díaz-Plaja y Vicens salieron con un programa de conferencias establecido, y ya anunciado previamente, que comportaba la preparación de las visitas o profundizaba en conocimientos adquiridos. También otros expedicionarios impartieron conferencias, como el profesor De las Barras de Aragón, el profesor Sas Murias o la catedrática Carmen Ambroj, única mujer entre los oradores. Todos los conferenciantes trataban de ajustar sus temas al itinerario y los intereses históricos del lugar y del momento. Los universitarios - a través del Dr. Ferrer y Cagigal - invitaron también a tres profesores puertorriqueños que viajaban en el Marqués de Comillas: se trataba de los profesores Pérez, Geigel y Cabrera. Lógicamente, las conferencias de Medicina, a cargo del Dr. Salvat y del director de la expedición, Ángel Ferrer y Cagigal, tuvieron una importancia capital, y, como en el caso de las sesiones humanísticas, guardaban estrecha relación con los lugares visitados: sobre todo hospitales y otras instalaciones sanitarias.

El diario de F. de las Barras nos ofrece preciosas noticias sobre este itinerario, que abundan en la vertiente didáctica del viaje. Por ejemplo, en la primera escala que hace el barco, en Valencia, Vicens Vives llevó a los alumnos al Museo de Prehistoria de la ciudad, organizado por Luis Pericot (Barras 1934: 11). También da cuenta del plan de conferencias. Según el autor del diario: «Lo fundamental de las conferencias eran los dos cursillos de siete conferencias, cada uno a cago de los señores Díaz Plaja y Vicens Vives [...]. En cuanto a las demás conferencias, no había ni podía haber un plan tan concreto ni se conocían los temas de muchas de ellas» (Barras 1934: 17).

Ya en la primera conferencia de Díaz-Plaja el autor del diario señala un aspecto que se va a repetir: la precepción que De las Barras tiene de las fricciones entre la cultura catalana y la castellana:

Aquella noche a las nueve dio su primera conferencia el señor Díaz-Plaja, que gustó bastante, pero sobre todo a los catalanes por tener alusiones no muy 
castellanas, por lo que a los no catalanes no nos sentó tan bien (Barras 1934: 18).

Nos informa también sobre el alto nivel de las conferencias:

Por la noche a las nueve dio el Sr. Vicens Vives su primera conferencia, que gustó bastante. Tanto el señor Díaz-Plaja como Vicens Vives son profesores que valen mucho y están a una altura superior al fin de vulgarización que se ha propuesto. Díaz-Plaja habla bien. Vicens también si lo hiciera en catalán, pero tiene una manifiesta dificultad para hablar en castellano. Se ve que piensa en catalán y traduce, el acento es bastante duro. Las conferencias de uno y otro están muy bien preparadas y muy bien hechas para divulgación entre personas de cierta cultura general; pero tienen un fuerte matiz catalanista. Ferrer, que es español hasta la médula, debe sufrir y encontrar dificultades en su gestión directora

En estas primeras páginas del diario el asunto de las conferencias es recurrente, lo que subraya el aspecto eminentemente formativo del viaje.

Sin perjuicio del interés cultural y científico, también había tiempo para los juegos, y los bailes, para tomar café mientras se oía música, para la piscina y para deportes propios de un barco. El jueves 2 de agosto escribe F. de las Barras:

En el menú del almuerzo, en que es costumbre anunciar el programa del día, se decía que aquella noche sería la comida de gala, seguida de baile [...]. Entre la comida y el baile, a las nueve de la noche, dio una conferencia el literato portorriqueño, y creo que notario de profesión, don Fernando Geigel [...] y estuvo muy bien (Barras 1934: 25)

Dice Guillermo Díaz-Plaja en una carta a sus padres fechada llegando a Puerto Rico, el 4 de agosto de 1934: «Se juega a saltar la cuerda, [...], a la rana y mucho ping pong. [...] Corriendo hacia abajo, a las "carreras de caballos", que se hacen con un cartón [por el] que avanzan - como en el parchís - con unos dados». También había cine, conciertos y mucho baile. Estas actividades les gustan, y aprovechan para ponerse las mejores galas que han encargado en sus ciudades de origen («Me he hecho un smoking blanco», comenta G. Díaz-Plaja en otra carta a sus padres) o que compran en los puertos. Así, la crucerista Concepción Taboada (en una carta a sus padres fechada en la Guayra el 8 de agosto de 1934) escribe: «Manolo - se refiere a su hermano- se compra un traje blanco, muy bonito, y un sombrero també bonito todo por ocho dólares. Los viajeros confiesan que les faltan horas para hacer tantas actividades. Y, sobre todo, que les falta capacidad para llegar a comer todo lo que la Compañía Transatlántica les ofrece. Explica Guillermo Díaz-Plaja en la misma carta del 4 de agosto de 1934: «Desayuno: unas lonchas de "jamón de york" o en dulce? Un par de huevos? Un embutido? Después: un vaso de lechecita, mermelada? mantequilla? Pastas? Croissant?. Después fruta: naranjas, peras, melocotones». La carta continúa describiendo las comidas en cantidad y en frecuencia ... 
Las actividades en el barco, la contemplación del mar, el descanso en las hamacas, los baños de sol en cubierta, la lucha contra el mareo o el calor o los vientos completaban una vida en la que no había tiempo de aburrirse. Los cruceristas tranquilizan a sus familias en las cartas, diciendo que todo va de maravilla y que están felices en el paraíso flotante. Escribe De las Barras el 28 de julio: «El tiempo magnífico sin ver un barco. En el nuestro dan un baile o mejor verbena en la terraza alta, rogándose a las señoras que asistieran con mantón de manila. Algunas que no lo tenían lo habían comprado en Las Palmas». (Barras 1934: 22).

\section{LA LLEGADA A LOS PUERTOS}

Pero la placentera actividad del barco se complementa con el laborioso movimiento de cada puerto al que llegaban. Las noticias que se recogen en el diario, en las cartas y en las crónicas mantienen siempre la misma tónica: comenzaban por una excelente recepción por autoridades académicas y / o culturales, muchas veces a cargo del Ministro Plenipotenciario de España. Por otra parte, los contactos con intelectuales, de los que se desprendían intercambios en forma de conferencias o de proyectos futuros. También había visitas que respondían a los intereses diversos de los cruceristas: podía ser en aulas universitarias, a hospitales, Casas de España o también, como en el caso de un grupo de ingenieros, visitas a refinerías como la Shell, o en el canal de Panamá, donde admiraron los aspectos más técnicos. Véase, por ejemplo, lo que dice F. de las Barras en su diario de la estancia en Puerto Rico:

Regresamos en parte por caminos diferentes y nos llevaron al Manicomio, que nos hicieron visitar al detalle y está dotado de los máximos adelantos. De allí nos llevaron hasta la Cárcel, también muy moderna, pero donde no entramos, y de allí al Dispensario Español, hospital modernísimo, admirablemente dotado y que también visitamos con todo detalle. Fuimos a continuación a la Universidad, situada en Río Piedras [...] donde dieron un lunch las señoritas alumnas en su residencia o departamento. Terminado esto salimos con dirección a un hotel, cuyo nombre no recuerdo, en que nos daba un banquete la Colonia Española pero con intervención de la Universidad pues presidió un catedrático (Barras 1934: 31-32).

En su diario, De las Barras ofrece un testimonio también de la transdisciplinariedad a que me he referido:

Había organizada una excursión para visitar la inmensa refinería de la Campsa, de petróleo [...]. Aunque esta visita era principalmente para técnicos, me incorporé $[\ldots . .$. . En la refinería permanecimos más de dos horas en que recorrimos aquella inmensa que podríamos llamar ciudad de tanques. [...]. Aunque completamente extraño a estos asuntos de ingeniería, estoy satisfecho de haber aprovechado esta ocasión única para visitar un establecimiento industrial que acaso es el primero del mundo en su clase

(Barras 1934: 43). 
De vez en cuando se encontraron con sorpresas inesperadas, en buena medida por cuestiones políticas relacionadas o bien con las dictaduras o con los nacionalismos. Por ejemplo, al terminar un banquete en Puerto Rico, unas palabras del orador fueron protestadas airadamente por uno de los asistentes, el Dr. Mangada. De las Barras lo cuenta así: «la intervención de Mangada produjo un gran revuelo con frases duras que no recuerdo. Creo que alguien se levantó para pegarle. [...] Parecía que iba terminar aquello como el Rosario de la Aurora, cuando el presidente se levantó, dijo unas palabras muy hábiles y se sosegaron los ánimos para que terminara el banquete con tranquilidad material» (Barras 1934: 33)

Quizá uno de los episodios más sonados fue el sucedido en Venezuela. Como afortunadamente contamos con varios testimonios epistolares, los cotejaremos con el diario de F. de las Barras. El asunto fue el siguiente: en la Guayra no pudieron cumplir el programa previsto porque la situación política de Venezuela no lo permitió. Llegó el Ministro de España y les comunicó el cambio, de Caracas a Macuto. Así lo explica el joven ingeniero Manuel Taboada en una carta fechada el 13 de agosto, llegando a Colón «nos dijo que teníamos que someternos a un plan». Y concluye diciendo: «No se han puesto del todo claros los motivos, pero la cosa fue que después de tener un programa proyectado para agasajarnos en Caracas, nos prohibieron el ir allí; se nos dijo que los estudiantes hubieran tomado nuestra visita [como excusa] para un movimiento contra el General Gómez, dictador del país».

De las Barras también señala que hubo varias teorías, pero al final se inclina por la siguiente explicación (fechada el 8 de agosto):

Después nos dijeron que no era nada de eso, sino que los estudiantes de Caracas nos tenían preparado un recibimiento magno y a la sombra de él una manifestación política y quizás un movimiento revolucionario: todo lo cual supo el Gobierno y fue causa de que nos impidieran ir a Caracas. Al saber la prohibición los estudiantes trataron de ir a la Guayra, y los detuvo en el camino la fuerza pública.

También nos dijeron que había habido sablazos y que había 52 detenidos.

El Gobierno, en efecto, no quería que desembarcáramos y sólo accedió a lo que se hizo a los ruegos del Ministro de España y el Rector, que se comprometieron personalmente a responder de que por nuestra parte no pasaría nada.

Parece que había dicho el Presidente Gómez que era preferible que hubiera algunos disgustados a que corriera la sangre por el camino de la Guayra a Caracas. Si las cosas llegaban hasta ese punto tenía razón.

A los viajeros que iban a quedar en el país no los dejaron subir a Caracas hasta que el Marqués de Comillas salió a la mar.

(Barras 1934: 40-41)

Una vez más, la idea de una expedición organizada por una universidad y un país plenamente democráticos causaba inquietud en ciertas posiciones políticas. En este sentido, el Dr. de las Barras de Aragón comenta en diversas oca- 
siones (pp. 63, 67 y 68, por ejemplo) que el Director del crucero tuvo que realizar una ímproba labor: «volvió Ferrer a tener que hacer equilibrios siempre por causa de las estridencias políticas de los oradores».

En otros casos los cruceristas comprueban in situ cuestiones que les sorprenden o les hacen reflexionar sobre los diversos aspectos coloniales. El Dr. de las Barras insiste en el trasfondo hispánico de un Puerto Rico americanizado. Otros no dejaron de lado el contacto con los movimientos nacionalistas. Más de cuarenta años después, escribe Guillermo Díaz-Plaja (1978: 317):

He vuelto muchas veces a deambular por las calles casi gaditanas del viejo San Juan, pero nunca he sentido la emoción de aquella primera visita, cuando, con un grupo de profesores y estudiantes, fuimos a visitar, en su modesto bohío de Río Piedras, al hombre que encabezaba un nacionalismo difícil y desesperado, que habría de llevarle a la cárcel y que se llamaba Pedro Albizu Campos. Y me parece que estoy viendo sus ojos inyectados en sangre, en la piel morena, gritando que iría a España a sacudirnos los hombros, porque no sabíamos comprender la dimensión su gesto.

Más adelante, en el mismo libro, el autor recuerda los contactos con los poetas de ritmos caribeños, con los académicos y escritores con quienes se relacionaron y también sus contactos con la poesía negra.

El mundo de la negritud fue, para muchos viajeros, un descubrimiento apasionante, en todas sus vertientes, no únicamente la literaria. Ven los diversos sedimentos de la población negra, y las diferentes proyecciones de cada país según los avatares de conquista y colonización. Así, la joven profesora de Historia Concepción Taboada, refiriéndose a Curaçao, escribe a sus padres en una carta fechada a bordo del Marqués de Comillas el 13 de agosto de 1934:

Fue este puerto la sorpresa del viaje, ya sabéis que es colonia holandesa, qué tipo de civilización más buena. [...]. Fuimos fisgoneando por las calles, y vimos las casas de los negros, limpias, bonitas, ellos haciendo vida de familia, la madre e hija cosiendo, el padre y el chico leyendo, algo tan diferente de otros sitios [...]. Los holandeses tienen este sistema de colonización, que no es el del español romántico que se une con ellos y da el tipo mestizo, ni el inglés o norteamericano que los veja o, mejor dicho, los suprime.

Las cuestiones culturales, históricas o antropológicas por un lado, y las científicas por otra no impedían que los viajeros universitarios sacaran el máximo jugo de todos y cada uno de los puertos. Sus anfitriones no se preocupaban únicamente de impartir conferencias o visitas técnicas. Les tenían preparadas también excursiones a la playa, banquetes, sesiones de baile, visitas a parques... también, en el poquísimo tiempo libre de que disponían, aprovechaban para ir a mercados y tocar y probar frutas tropicales, comprar recuerdos, observar el precio de vestimenta, las maneras de hacer de la gente, hacerse fotografías... 


\section{LLEGADA A NUEVA YORK Y REGRESO A ESPAÑA}

Después de las ciudades centroamericanas y de la cultura caribeña, Nueva York los deslumbra en un sentido totalmente diferente. Escribe Manuel Taboada en una carta sus padres fechada el 27 de agosto de 1934: «Yo os puedo garantizar que a pesar de haber visto la entrada en New York muchísimas veces en películas, con su estatua de la Libertad y los rascacielos, puentes monumentales, etc. me impresiona profundamente». Guillermo Díaz-Plaja impartió una conferencia en la Casa de España de Columbia University, y, sin embargo, aún tuvieron tiempo de ir a Coney Island, subir al Empire State, West Point... francamente, parece que multiplicaban el tiempo!

Hasta para el experimentado viajero que fue Francisco de las Barras, la llegada a Nueva York (el 25 de agosto de 1934) marca un hito, que refleja así en su diario:

Lo más interesante del paseo nocturno por la ciudad fue el aspecto de los rascacielos de noche y la profusión inmensas de luces en la parte céntrica, donde está reunido el máximo número de teatros en Broadway, en Times Square y cruce de las calles 38 a 60 .

El parque de atracciones donde estuvimos era inmenso, con un verdadero derroche de luz y una gran variedad de tubos de risa, montañas rusas, plataformas movibles etc. etc. El público era muy numeroso.

(Barras 1934: 72)

Lo asombroso es la visita de la enorme ciudad de más de nueve millones de habitantes que siguen aumentando. La visita de Nueva York por encima resulta extraña; hay una diferencia enorme entre ella y todo lo que uno ha visto, pues los rascacielos, que son mucho más numerosos de lo que yo creía, le dan un carácter propio y único. Andando por abajo el aspecto general prescindiendo de los rascacielos recuerda bastante a Londres.

Es muy difícil conservar de un modo concreto las impresiones de aquel día. Nos llevaron un momento a ver una magnífica estación de ferrocarriles, creo que central, llamada de Pensilvania, pasamos a Brooklyn por el célebre puente, de que tanto se habló al tiempo de su construcción, porque se le dio una altura que permitía pasar por debajo los buques de vela de mayor arboladura; cruzando un barrio de negros y otro de judíos y salimos de la ciudad llegando hasta la puerta de la prisión de Sing-Sing, que no visitamos.

Recorrimos las orillas del río Hudson entre paisajes hermosísimos, primero de parques y luego de montañas, cubiertas de bosque natural que como de clima templado recuerda por su aspecto la vegetación europea.

(Barras 1934: 73).

Después del viaje a Nueva York, el diario de Francisco de las Barras se hace cada vez más telegráfico y apenas da detalles sobre el regreso. Un año después, en 1935 su autor volverá a embarcarse en otro crucero organizado de 
nuevo por el Dr. Ángel Ferrer Cagigal, esta vez con destino a Extremo Oriente, y también llevará un diario (que tampoco ha sido editado). Ese mismo año Francisco de las Barras impartió una conferencia (a partir de las notas de su diario) sobre el Crucero de 1934 en la Sociedad Geográfica donde dijo lo siguiente: «Resulta de lo dicho que el Crucero Trasatlántico de la Universidad de Barcelona ha sido un éxito indudable, que probablemente se completará con alguna excursión semejante hecha por americanos a España» (Barras 1935a: 23). En conclusión, el crucero de 1934 fue un verdadero éxito y supuso un nuevo modo de enfocar el aprendizaje universitario, y el diario del Dr. Francisco de las Barras de Aragón constituye un testimonio valiosísimo para conocerlo de primera mano.

\section{BIBLIOGRAFÍA}

AA.VV. (2006) Querido amigo, estimado maestro: Cartas a Guillermo DíazPlaja, Edición a cargo de Ana Díaz-Plaja, Jordi Amat Fusté y Blanca Bravo Cela. Introducción de Anna Caballé, Barcelona, Publicacions de la Universitat de Barcelona-Reial Acadèmia de Bones Lletres de Barcelona.

Barras y de Aragón, F. de las (1934) Primer crucero universitario de Barcelona (diario mecanoscrito inédito).

- (1935 a) «El crucero trasatlántico de la Universidad de Barcelona». Conferencia por D. Francisco de las Barras y de Aragón, en la Sociedad Geográfica, el 11 de marzo de 1935 (inédito).

- (1935 b) «El crucero trasatlántico de la Universidad de Barcelona», Boletín de la Sociedad Geográfica Nacional, n. ${ }^{\circ}$ LXXV (junio 1935), pp. 323-334.

Biagi, P. di (2006) «Los CIAM de camino a Atenas. Espacio habitable y ciudad funcional», en. Landrove, S. (ed.); Puente, M. trad. El G.A.T.C.P.A.C. y su tiempo: política, cultura y arquitectura en los años treinta $=O$ G.A.T.C.P.A.C. e o seu tempo: política, cultura e arquitectura nos anos trinta: Actas" / V Congresso Fundação Docomomo Ibérico, Barcelona, 26-29/10/2005. Barcelona, Fundación Docomomo Ibérico, pp. 135-148.

Consultable en: http://www.planum.net/download/dibiagi-ciam-art-spagn-pdf (fecha de consulta: 26-3-2018).

Díaz-Plaja, A. (2016) «Las mujeres se embarcan hacia el futuro: las cruceristas de 1934», Temps d'Educació, 51, pp. 107-122.

Díaz-Plaja, A. + Jiménez León, M. (2015) «"L’altre” creuer universitari. L'expedició a Amèrica del 1934», L'Avenç, 409 (febrer), pp. 22-28.

Díaz-Plaja, G. (1935) Cartes de navegar. Barcelona, Catalònia [Segunda edición: Argentona, L’Aixernador, 1992].

- (1966) Memoria de una generación destruida: 1930-1936, Barcelona, Delos-Aymá. Prólogo de Julián Marías.

- (1978) Retrato de un escritor. Barcelona, Editorial Pomaire. 
Esteve Gálvez, F. (2009) En el contorno de las aguas luminosas. El crucero universitario de 1933. «Perfil biobibliográfico de Francisco Esteve Gálvez», a cargo de José Ramón Magdalena Nomdedéu. Zaragoza, Institución Fernando el Católico (C.S.I.C.), Excma. Diputación Provincial de Zaragoza.

Gracia Alonso, F. y Fullola i Pericot, J. M. (2006) El sueño de una generación. El crucero universitario por el Mediterráneo de 1933. Barcelona, Publicacions i Edicions de la Universitat de Barcelona.

Jiménez León, M. (2016a) «Dos diarios inéditos de los cruceros organizados por la Universidad Autónoma de Barcelona en 1934 y 1935», Temps d'Educació, 51, pp. 95-106.

- (2016b) «Un ejemplo de transversalidad académica y multimodal: la guía didáctica para la exposición sobre el crucero de 1934» en Díez Mediavilla, A.; Brotons Rico, V.; Escandell Maestre, D. y Rovira Collado, J. (eds.): Aprendizajes plurilingües y literarios. Nuevos enfoques didácticos, Alacant pp. 487-494.

Marías, J. (2011) Diario de un viaje a Oriente. Edición de Daniel Marías y Francisco Javier Jiménez. Epílogo de Javier Marías. Madrid, Páginas de Espuma.

Matías-Guiu Guia, X. + Martí Laborda, R. M. (1981) Estudio biográfico del profesor Ángel A. Ferrer Cagigal. Estudio inédito, que obtuvo el tercer lugar en el XII Premio URIACH de Historia de la Medicina.

Merino Escobar, M. (2016) «Cruceristas en el año 1934: otro descubrimiento de América», Temps d'Educació, 51, pp. 123-135.

Sotelo Vázquez, Adolfo (2014) De Cataluña y España. Relaciones culturales y literarias (1868-1960), Barcelona, Publicacions i Edicions de la Universitat Barcelona.

Valiente Romero, A. (2007) Francisco de las Barras de Aragón en la Sevilla intersecular. Sevilla, Ateneo de Sevilla-Universidad de Sevilla.

- (2013) «El legado de Francisco de las Barras de Aragón en la Biblioteca de la Universidad de Sevilla. Aproximación a la procedencia de sus ejemplares», en Peñalver Gómez, Eduardo (coord.): Fondos y procedencias: Bibliotecas en la Biblioteca de la Universidad de Sevilla. Sevilla, Universidad de Sevilla, Secretariado de Publicaciones.

http://expobus.us.es/tannhauser/ftp/file/procedencias/2012_Sala5_09_ Introduccion.pdf (fecha de consulta: 26-3-2018).

Vilafranca, I. (2009) «La influència del krausisme a Catalunya». Temps d'Educació, 37, p. 39-50. 
